\title{
Response of the solar atmosphere to magnetic field evolution in a coronal hole region
}

\author{
S. H. Yang ${ }^{1}$, J. Zhang ${ }^{1}$, C. L. Jin ${ }^{1}$, L. P. $\operatorname{Li}^{1}$, and H. Y. Duan ${ }^{2}$ \\ 1 Key Laboratory of Solar Activity, National Astronomical Observatories, Chinese Academy of Sciences, Beijing 100012, PR China \\ e-mail: [shuhongyang;zjun]@ourstar.bao.ac.cn \\ 2 Jiangsu Sopo Corporation Group Ltd., Zhenjiang 212006, PR China
}

Received 15 July 2008 / Accepted 14 April 2009

\begin{abstract}
Context. Coronal holes $(\mathrm{CHs})$ are deemed to be the sources of the fast solar wind streams that lead to recurrent geomagnetic storms and have been intensively investigated, but not all the properties of them are well known.

Aims. We study the response of the solar atmosphere to the magnetic field evolution in a $\mathrm{CH}$ region, such as magnetic flux emergence and cancellation for both network (NT) and intranetwork (IN) regions.

Methods. We study an equatorial CH observed simultaneously by HINODE and STEREO on July 27, 2007. The HINODE/SP maps are adopted to derive the physical parameters of the photosphere and to investigate the magnetic field evolution and distribution. The $\mathrm{G}$ band and $\mathrm{Ca}$ II $\mathrm{H}$ images with high tempo-spatial resolution from HINODE/BFI and the multi-wavelength data from STEREO/EUVI are utilized to study the corresponding atmospheric response of different overlying layers.

Results. We explore an emerging dipole located at the $\mathrm{CH}$ boundary. Mini-scale arch filaments (AFs) accompanying the emerging dipole were observed with the Ca II H line. During the separation of the dipolar footpoints, three AFs appeared and expanded in turn. The first AF divided into two segments in its late stage, while the second and third AFs erupted in their late stages. The lifetimes of these three AFs are 4, 6,10 min, and the two intervals between the three divisions or eruptions are 18 and 12 min, respectively. We display an example of mixed-polarity flux emergence of IN fields within the $\mathrm{CH}$ and present the corresponding chromospheric response. With the increase of the integrated magnetic flux, the brightness of the Ca II $\mathrm{H}$ images exhibits an increasing trend. We also study magnetic flux cancellations of NT fields located at the $\mathrm{CH}$ boundary and present the chromospheric and coronal response. We notice that the brighter regions seen in the $171 \AA$ images are relevant to the interacting magnetic elements. By examining the magnetic NT and IN elements and the response of different atmospheric layers, we obtain close positive linear correlations between the NT magnetic flux densities and the brightness of both $\mathrm{G}$ band (correlation coefficient 0.85 ) and $\mathrm{Ca}$ II $\mathrm{H}$ (correlation coefficient 0.58 ).
\end{abstract}

Key words. Sun: magnetic fields - Sun: evolution - Sun: atmosphere - Sun: filaments

\section{Introduction}

Coronal holes $(\mathrm{CHs})$ are regions on the Sun where the magnetic fields are dominated by one magnetic polarity and the magnetic lines are open to interplanetary space (Bohlin 1977). These unipolar structures are presumed to originate at about $0.7 R_{\odot}$ distance from the solar center (Stepanian 1995). CHs were identified as early as in 1951 by Waldmeier (1951) from the earliest photos of the solar corona. Observed with X-ray (Underwood \& Muney 1967) and EUV lines (Reeves \& Parkinson 1970), they appear as dark void areas due to their lower densities and lower temperatures compared with that of the quiet Sun (QS) (Munro \& Withbroe 1972; Harvey 1996). But if observed with the He I $10830 \AA$ line, they are brighter areas (Zirker 1977; Harvey \& Sheeley 1979). CHs are usually classified into three different categories according to their locations and lifetimes: polar, nonpolar (isolated), and transient (Harvey \& Recely 2002). Polar $\mathrm{CHs}$ can persist for several years, non-polar ones always for many solar rotations, while transient ones persist for only several days. They are deemed to be the sources of the fast solar wind streams that lead to recurrent geomagnetic storms (Krieger et al. 1973; Tsurutani \& Gonzalez 1987; Crooker \& Cliver 1994; Cranmer 2002; Tu et al. 2005).

A large amount of research concerning $\mathrm{CHs}$ has been carried out in recent years. These studies examine nearly all the properties of $\mathrm{CHs}$, such as positional distribution and periodic variation (Belenko 2001; Maravilla et al. 2001; Harvey \& Recely 2002; Bilenko 2002, 2004; Hofer \& Storini 2002; Mahajan et al. 2002; Temmer et al. 2007), magnetic field structure (Meunier 2005; Wiegelmann et al. 2005; Tian et al. 2008), magnetic field evolution (Wang \& Sheeley 2004; Yamauchi et al. 2004; Zhang et al. 2006), temperature variation (Patsourakos et al. 2002; Wilhelm 2006; Zhang et al. 2007a), element abundance (Feldman \& Laming 2000; Laming \& Feldman 2003), Doppler velocity (Raju et al. 2000; Stucki et al. 2000, 2002; Jordan et al. 2001; Wilhelm et al. 2002; Kobanov et al. 2003; Xia et al. 2003, 2004; Akinari 2007; Teplitskaya et al. 2007), fast solar wind (Giordano et al. 2000; Hackenberg et al. 2000; Patsourakos \& Vial 2000; Wilhelm et al. 2000; Teriaca et al. 2003; Zhang et al. 2002, 2003, 2005a; Janse et al. 2007) and waves in CH (Moran 2003; Zhang 2003; Markovskii \& Hollweg 2004; O'Shea et al. 2005, 2006, 2007; Zhang et al. 2005b; Dwivedi et al. 2006; Kobanov \& Sklya 2007; Srivastava et al. 2007; Wu et al. 2007).

According to previous studies, $\mathrm{CHs}$ are not absolutely unipolar and there exist many closed coronal loops (eg. Levine 1977; Zhang et al. 2006). The magnetic network (NT; Leighton et al. 1962) elements are believed to consist of both low-lying loops and large-scale open magnetic funnels (Dowdy et al. 1986; Dowdy 1993), while the intranetwork (IN; Livingston \& Harvey 1975) only contributes low-lying loops 
(Wiegelmann \& Solanki 2004; Wiegelmann et al. 2005). In equatorial $\mathrm{CHs}$, the chromosphere and transition region are highly structured. Their structures are similar to those in the QS region (Warren \& Winebarger 2000; Feldman et al. 2001). Above the upper transition region, if observed with X-ray or EUV lines, most of the structures disappear and the corona becomes much darker and more homogeneous than in the QS, except for some bright points (Xia et al. 2004).

Magnetic flux emergence and cancellation are the main forms of magnetic field evolution in the Sun (Zhang et al. $1998 \mathrm{a}-\mathrm{c})$. In an emerging flux region, new emerging dipoles may lead to the formation of arch filament systems (AFSs). AFSs were first studied by Bruzek (1967). Since then, their properties, such as size, shape, lifetime, evolution, structure and so on, have been extensively investigated (Bruzek 1969; Weart 1970; Frazier 1972; Chou \& Zirin 1988; Alissandrakis et al. 1990; Georgakilas et al. 1990; Tsiropoula et al. 1992; Chou 1993). The reconnection occurring at $\mathrm{CH}$ boundaries is crucial to the evolution and rigid rotation of CHs (Wang et al. 1996; Kahler \& Hudson 2002).

There is always a good correlation between the QS magnetic NT elements and the chromospheric structures since the magnetic NT features are always bright in the Ca II line (Leighton 1959; Frazier 1970; Skumanich et al. 1975; Stenflo \& Harvey 1985; Zirin 1988; Rezaei et al. 2007). Although the studies from Sivaraman \& Livingston (1982) and Sivaraman et al. (2000) reveal that magnetic concentrations play a major role in the formation of Ca II K line IN elements and that there is a one-to-one relationship between $\mathrm{K}$ line $\mathrm{IN}$ bright elements and magnetic features, some other authors (eg. Remling et al. 1996; Steffens et al. 1996) report that there is no correlation between smallscale magnetic elements and the location of bright $\mathrm{Ca} \mathrm{K}_{2 v}$ IN elements. Nindos \& Zirin (1998) quantitatively studied the relation between the intensity of Ca II K line bright features and the intensity of the associated magnetic elements in the QS. They found that there is an almost linear correlation between the K-line intensities and the absolute values of the magnetic field strength for the stronger NT elements, while this correlation disappears for the weaker magnetic elements. Lites et al. (1999) investigated the QS IN and found no direct correlation between the presence of magnetic features with an apparent flux density above $3 \mathrm{Mx} \mathrm{cm}^{-2}$ and the occurrence of $\mathrm{H}_{2 v}$ brightenings. They also found no correspondence between $\mathrm{H}_{2 v}$ grains and the horizontal-field IN features. Worden et al. (1999) found only a random correspondence between bright cell grains and regions of IN magnetic flux as seen in H I Ly $\alpha$ and $160 \mathrm{~nm}$ lines. Recently, Rezaei et al. (2007) investigated the relationship between the photospheric magnetic field and the emission of the mid-chromosphere and found that the emission in the NT is correlated with the magnetic flux density, while there is no correlation between the integrated emission of $\mathrm{Ca}$ core-line and the magnetic flux density in the IN.

Due to the restrictions of observations, not all the characteristics of CHs are well known. Several newly launched spacebased instruments, especially HINODE (Kosugi et al. 2007), have provided unprecedentedly high spatial and temporal resolution data, which is extremely important in investigating the fine structures in detail. Furthermore, multi-passband images from the Solar Terrestrial Relations Observatory (STEREO; Howard et al. 2008; Kaiser et al. 2008) give us plentiful information about the solar atmosphere. These data can be used together to study the magnetic field evolution and the response of the overlying atmosphere, which will be helpful for us to understand the physical mechanism of coronal heating and solar wind acceleration.
Table 1. Data sets used in this study.

\begin{tabular}{ccccc}
\hline \hline Data set & Observation & $\begin{array}{c}\text { Cadence } \\
(\mathrm{min})\end{array}$ & $\begin{array}{c}\text { Pixel size } \\
(\operatorname{arcsec})\end{array}$ & $\begin{array}{c}\text { FOV } \\
\left(\operatorname{arcsec}^{2}\right)\end{array}$ \\
\hline I & HINODE/SP & $32^{a}$ & 0.32 & $151.14 \times 162.30$ \\
II & HINODE/Gband & 2 & 0.11 & $223.15 \times 111.58$ \\
& HINODE/Ca II H & 2 & 0.11 & $223.15 \times 111.58$ \\
III & STEREO/304 & 10 & 1.59 & full disk \\
& STEREO/171 & 2.5 & 1.59 & full disk \\
& STEREO/195 & 10 & 1.59 & full disk \\
& STEREO/284 & 20 & 1.59 & full disk \\
\hline
\end{tabular}

${ }^{a}$ Scan time for one SP map.

Here, in order to study magnetic flux emergence, cancellation, distribution, and the atmospheric response of different overlying layers in $\mathrm{CHs}$, we investigate an equatorial $\mathrm{CH}$ of predominantly negative polarity. In Sect. 2, we introduce the observations and data analysis. In Sect. 3, we give some typical examples of small-scale magnetic field evolution and the corresponding atmospheric response, as well as the relationship between the magnetic intensities and the brightness of the overlying solar atmosphere. The discussion and conclusions are presented in Sect. 4.

\section{Observations and data analysis}

The observations were carried out on July 27, 2007, using the Solar Optical Telescope (SOT; Ichimoto et al. 2008; Shimizu et al. 2008; Suematsu et al. 2008; Tsuneta et al. 2008) on board HINODE, the Extreme Ultra Violet Imager (EUVI; Howard et al. 2008) telescope of the Sun-Earth Connection Coronal and Heliospheric Investigation (SECCHI; Howard et al. 2008) instrument aboard STEREO, the Michelson Doppler Imager (MDI; Scherrer et al. 1995) and the Extreme-ultraviolet Imaging Telescope (EIT; Delaboudinière et al. 1995) onboard the Solar and Heliospheric Observatory (SOHO; Domingo et al. 1995).

The data sets used in this study are summarized in Table 1. The spectro-polarimeter (SP; Lites et al. 2001) in HINODE/SOT provides observations in four modes: normal, fast, dynamics and deep maps. Five sets of HINODE/SP maps adopted here were observed in fast map mode between 01:36 UT and 05:58 UT. They are all centered at about $11^{\circ} \mathrm{W}$ and $1^{\circ} \mathrm{S}$ and cover a large fraction of the $\mathrm{CH}$ area. Each map is constructed from $512 \times 512$ Stokes $(I, Q, U$ and $V$ ) profiles of the photospheric Fe I $6301.5 \AA$ and $6302.5 \AA$ lines with a spectral sampling of $21.5 \mathrm{~m} \AA$. The noise level in the continuum polarization is about $1.5 \times 10^{-3} I_{\mathrm{c}}$. For the observation of each spectrograph slit, the integrated exposure time is $3.2 \mathrm{~s}$. The scan direction is along the east-west direction with a scan step of 0.'295.

By using the Stokes spectrum inversion code based on the assumption of Milne-Eddington atmospheres (Yokoyama, T. private communication), we have successfully derived numerous physical parameters from the raw data, such as the three components of magnetic fields (the field strength $B$, the inclination angle $\gamma$, and the azimuth angle $\phi$ ), the stray light fraction $\alpha$, and the Doppler velocity $V_{\text {los }}$. Here, $\gamma$ is the angle between the vector magnetic field $\boldsymbol{B}$ and the LOS direction, and $\phi$ is the angle from the east-west direction to the projection of $\boldsymbol{B}$ on the plane perpendicular to the LOS direction. Then the vector magnetic field $\boldsymbol{B}$ is shown by the LOS field $(1-\alpha) B \cos \gamma$ and the transverse field $(1-\alpha)^{1 / 2} B \sin \gamma$. The Doppler shifts are derived from the Fe I $6302.5 \AA$ Stokes I profiles and averaged over the whole field of view (FOV) of the SP maps. 


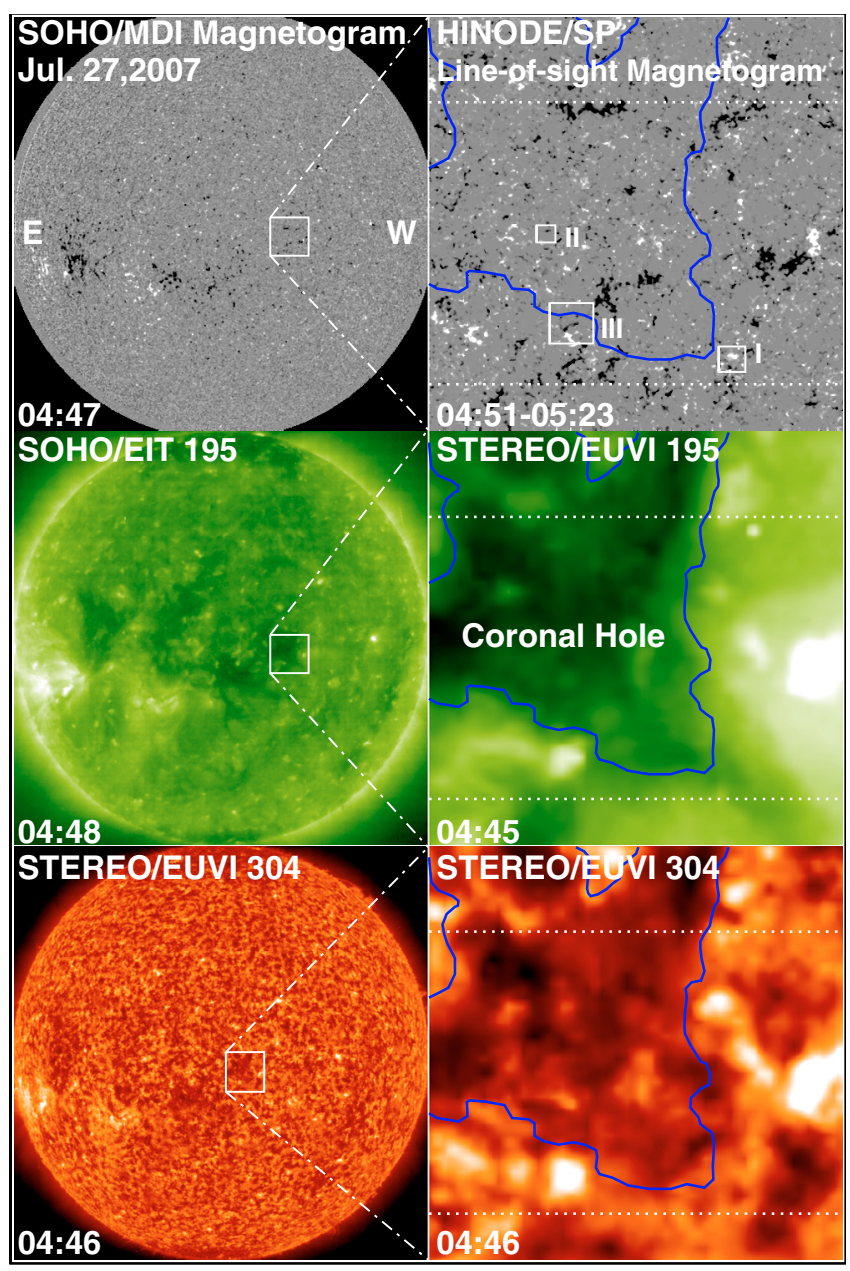

Fig. 1. SOHO/MDI full-disk magnetogram (top left), SOHO/EIT $195 \AA$ image (middle left), and STEREO/EUVI $304 \AA$ image (bottom left). Three windows in the three full-disk images outline the FOV of HINODE/SP magnetograms. The right column shows a line-of-sight magnetogram from $H I N O D E / S P$, a $195 \AA$ image and a $304 \AA$ image from STEREO from top to bottom. The magnetograms from MDI and SP are displayed with $(-50 \mathrm{G}, 50 \mathrm{G})$ and $(-80 \mathrm{G}, 80 \mathrm{G})$ scales, respectively. The blue curves delineate the $\mathrm{CH}$ boundary derived from the STEREO/EUVI $284 \AA$ image obtained at 04:46 UT, while the dotted lines indicate the FOV of the $\mathrm{G}$ band and $\mathrm{Ca}$ II $\mathrm{H}$ images. The rectangles in the top-right image delineate the regions ("I", "II", "III") studied.

$\mathrm{G}$ band and $\mathrm{Ca}$ II $\mathrm{H}$ images obtained by the Broadband Filter Imager (BFI; Kosugi et al. 2007) are also employed from HINODE. Their FOV only covers part of the SP maps (shown with the dotted lines in Fig. 1). We adopt the EUVI data from satellite A of STEREO. The EUVI telescope observed the full Sun in four spectral channels (304 A, $171 \AA, 195 \AA$ and $284 \AA$ ). The HINODE/BFI and STEREO/EUVI images used here are selected according to the scanning time of the SP maps.

The images are all prepared by applying standard processing routines, including flat field correction, dark current and pedestal subtraction, bad camera pixel correction, camera readout error correction, cosmic ray removal, et al.. Then we coalign all the images and SP maps carefully. Since both G band and $\mathrm{Ca}$ II $\mathrm{H}$ observations were taken with fixed pointing, first we co-align these images with each other. The bright features of $\mathrm{Ca}$ II $\mathrm{H}$ line images are highly coincident with the underlying magnetic field features both in location and shape, especially for NT elements (Warren \& Winebarger 2000; Feldman et al. 2001;

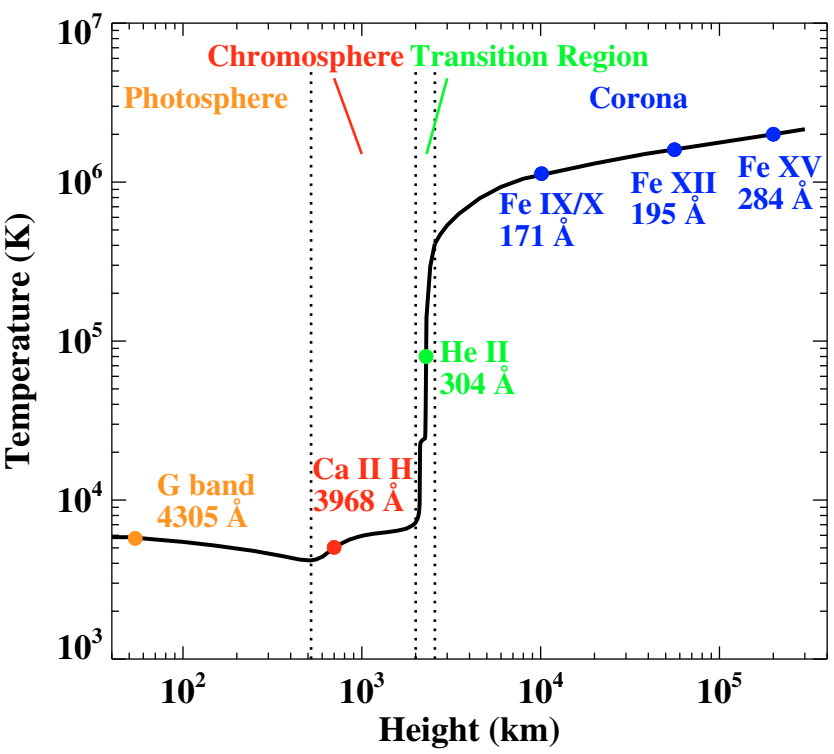

Fig. 2. Variation of the temperature versus the height above the $\tau_{5000}=1$ (optical depth unity in the continuum at $5000 \AA$ ) surface. The $\mathrm{G}$ band and $\mathrm{Ca}$ II $\mathrm{H}$ lines are emitted respectively in the lower photosphere and lower chromosphere, and their mean formation heights $(54 \mathrm{~km}$ and $700 \mathrm{~km}$, respectively) are all marked with filled circles in this figure. The peak formation temperatures of the He II (304 $\AA$ ) line emitted in the transition region, and Fe IX/X (171 ̊), Fe XII (195 ̊), Fe XV (284 ̊) lines which are all emitted in the inner solar corona, are $80000 \mathrm{~K}$, 1.3 MK, 1.6 MK, 2.0 MK, respectively. They also marked with filled circles in this figure.

Xia et al. 2004), so we use the bright points to co-align the $G$ band and $\mathrm{Ca}$ II $\mathrm{H}$ images with the LOS magnetograms. For the co-alignment between the STEREO images and the SP maps, we use the $S O H O / M D I$ and $S O H O / E I T$ images. The LOS magnetograms (SP maps) from HINODE are co-aligned with the MDI full-disk magnetograms. Since the EIT and MDI images are from the same satellite and they can be co-aligned easily, the SP maps can be co-aligned with the EIT images without difficulty. Then the STEREO $195 \AA$ images are co-aligned with the SOHO $195 \AA$ images according to their bright features. After that, the STEREO $195 \AA$ images (also $304 \AA$, $171 \AA$ and $284 \AA$ ) were co-aligned with the SP maps.

We determine the $\mathrm{CH}$ boundary with the brightness gradient method developed by Shen et al. (2006). In an EUV $284 \AA$ image, each pixel has its own recorded brightness, $b$. For any given value of $b$, we can plot the contour and then calculate the area, $A$, enclosed by each contour. The derivation $f=\delta b / \delta A$ is used to determine the boundary of a $\mathrm{CH}$. The $\mathrm{CH}$ boundary is where $f=f_{\text {max }}$.

Figure 1 shows a $S O H O / \mathrm{MDI}$ magnetogram (top left), a SOHO/EIT $195 \AA$ image (middle left) and a STEREO/EUVI $304 \AA$ image (bottom left). Three windows in the three full-disk images outline the FOV of HINODE/SP magnetograms. The right column shows respectively a LOS magnetogram from HINODE, a $195 \AA$ image and a $304 \AA$ image from STEREO. In order to improve the image contrast to visualize the fine structures better, a log-logarithmic scale is applied to the STEREO images presented in all the figures.

Figure 2 shows the typical variation of the temperature with the height of the solar atmosphere above the $\tau_{5000}=1$ surface (Reeves et al. 1977; Vernazza et al. 1981). The G band and $\mathrm{Ca}$ II $\mathrm{H}$ lines are emitted respectively in the photosphere 


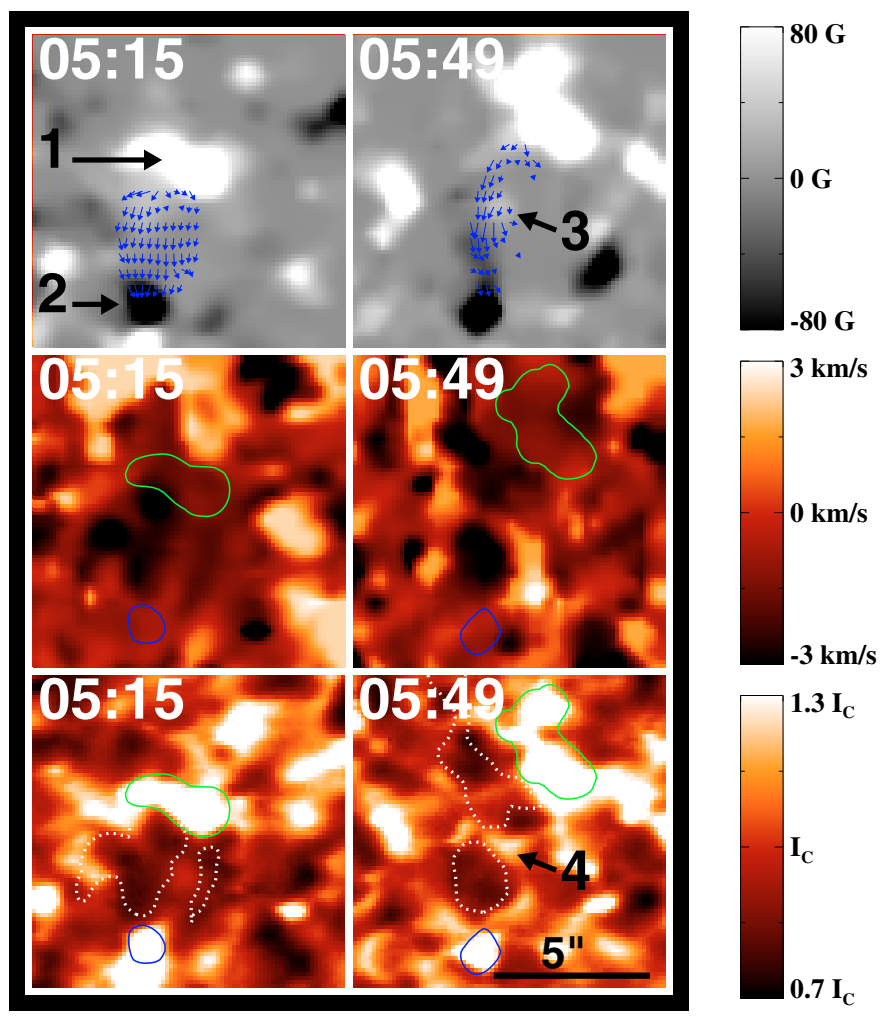

Fig. 3. LOS magnetograms (region "I" in Fig. 1) at different times (the upper panels) with an emerging dipole indicated by arrow "1" (positive) and arrow " 2 " (negative), corresponding Dopplergrams (the middle panels) and $\mathrm{Ca}$ II $\mathrm{H}$ images (the bottom panels). The green and blue curves are contours of the positive $(+80 \mathrm{G})$ and negative $(-80 \mathrm{G})$ elements, respectively. The blue arrows show the transverse fields between the dipolar elements, while the dotted lines are contours of the Doppler blue shift $\left(-2.0 \mathrm{~km} \mathrm{~s}^{-1}\right)$. Arrows " 3 " and " 4 " denote the place of a newly formed magnetic concentration. The $I_{\mathrm{c}}$ (same as in Figs. 4, 6, and 7) marked on the color bar represents the average value of $\mathrm{Ca}$ II $\mathrm{H}$ intensities in the FOV of $\mathrm{Ca}$ II $\mathrm{H}$ images.

and lower chromosphere, and their mean formation heights are $54 \mathrm{~km}$ (Carlsson et al. 2004) and $700 \mathrm{~km}$ (Beck et al. 2008), respectively. The He II (304 $\AA$ ) line is emitted in the transition region, while the Fe IX/X (171 ̊), Fe XII (195 ^), Fe XV (284 ̊) lines are all formed in the inner solar corona. The peak formation temperatures of these four passbands are $80000 \mathrm{~K}, 1.3 \mathrm{MK}$, 1.6 MK, 2.0 MK, respectively (Delaboudinière et al. 1995). All these mean heights or peak temperatures are marked with filled circles in this figure. We can see clearly that these different lines (or images) represent different heights (or layers) of the solar atmosphere. This means that, in order to study the response of the different overlying layers to the magnetic field evolution and distribution, we can study the structures seen in different pass-band images.

All the spectral images are separately composed into new integrated images with time slices synchronised with the time sequences of SP scanning, so as to maintain their temporal correspondence.

\section{Results}

\subsection{Magnetic flux emergence at the $\mathrm{CH}$ boundary}

Magnetic flux emergence is the main form of magnetic field evolution. Here, we study an emerging dipole at the $\mathrm{CH}$ boundary using the data from HINODE/SOT.
Figure 3 shows the region "I" in Fig. 1 in an expanded view. Two LOS magnetograms (the upper panels) display an emerging dipole indicated by arrows " 1 " (positive) and " 2 " (negative). The blue arrows show the transverse magnetic fields between the dipolar elements, while the dotted lines are contours of the Doppler blue shift $\left(-2.0 \mathrm{~km} \mathrm{~s}^{-1}\right)$. We can see that the transverse fields between the dipolar elements point from the positive element to the negative one, and in the narrow zones where the transverse fields lie, there are strong blue shifts relative to the surrounding parts. In the bottom panels, the darker features on the Ca II H images mainly coincide with the stronger blue shift places. Note that the break in the Doppler structure at 05:49 UT (indicated by arrow "4") is caused by a newly emerging flux in the corresponding place (pointed by arrow " 3 ").

The chromospheric response to the emergence of this dipole is shown in Fig. 4. The sequence of Ca II H images shows the development process of arch filaments (AFs). The dipolar elements (indicated by arrows " 1 " and " 2 ") at 05:15 UT and 05:49 UT have been contoured to the temporally nearest panels. Three AFs were observed in turn. Each column shows the development of one AF. The first AF appeared at 05:07 UT and then expanded. At 05:09 UT, the AF looked like a dark absorption region. This AF expanded and then divided into two segments. At 05:15 UT, the two segments connecting two brighter patches were much obvious. Comparing with SP magnetograms, we noticed that the bright patches in Ca II were co-spatial with the concentrations of the magnetic field of both polarities. The two segments persisted for several minutes without obvious changes in size. At 05:23 UT, another similar AF appeared at the same location as the previous one and began to grow. Two minutes later, the AF was much more obvious, as seen in the image labeled " $05: 25$ ". The AF erupted suddenly (at 05:29 UT) and dissipated quickly, so that it could not be distinguished from the background in the next image (at 05:31 UT). Arrow " 3 " denotes the main body of this AF (described with the thin dotted line). In a similar way as in the middle column, the right shows the erupting process of the last AF. It emerged following the eruption of the former one, continued to expand and erupted suddenly at 05:41 UT.

We measure the width (bi-head arrow in Fig. 4) of the AFs in each Ca II image and show the temporal evolution of the measured width with the filled circles in Fig. 5. When the AF divided or no AF could be distinguished from the background, the width was set to zero and represented with unfilled circles. The solid line "1" ("2"/“"3") connecting the filled circles represents the visible width variation of an AF, while dotted lines indicate the invisible stages. The lifetimes of these three AF are 4, 6, 10 min and the two intervals between the three divisions or eruptions are 18 and $12 \mathrm{~min}$, respectively. Also plotted in this figure is the total unsigned magnetic flux (connected with a dashed line) of the dipole, increasing from $7.48 \times 10^{18} \mathrm{Mx}$ to $1.22 \times 10^{19} \mathrm{Mx}$ in 34 min.

\subsection{Magnetic flux emergence in the $\mathrm{CH}$}

Except for the obvious dipolar emergence, we also find that many magnetic fluxes emerge in the form of mixed-polarity clusters. Figure 6 shows an example of IN flux emergence (region "II" in Fig. 1) in the $\mathrm{CH}$. The left column shows LOS magnetograms, while the right one consists of Ca II H images. Green and blue contours represent the positive and negative magnetic elements at $+15 \mathrm{G}$ and $-15 \mathrm{G}$ levels. The total unsigned magnetic flux of this region increased from $5.17 \times$ $10^{17} \mathrm{Mx}(01: 44 \mathrm{UT})$ to $1.24 \times 10^{18} \mathrm{Mx}(02: 18 \mathrm{UT})$, then reached $2.47 \times 10^{18} \mathrm{Mx}(03: 21 \mathrm{UT})$. On the other hand, we calculate the 

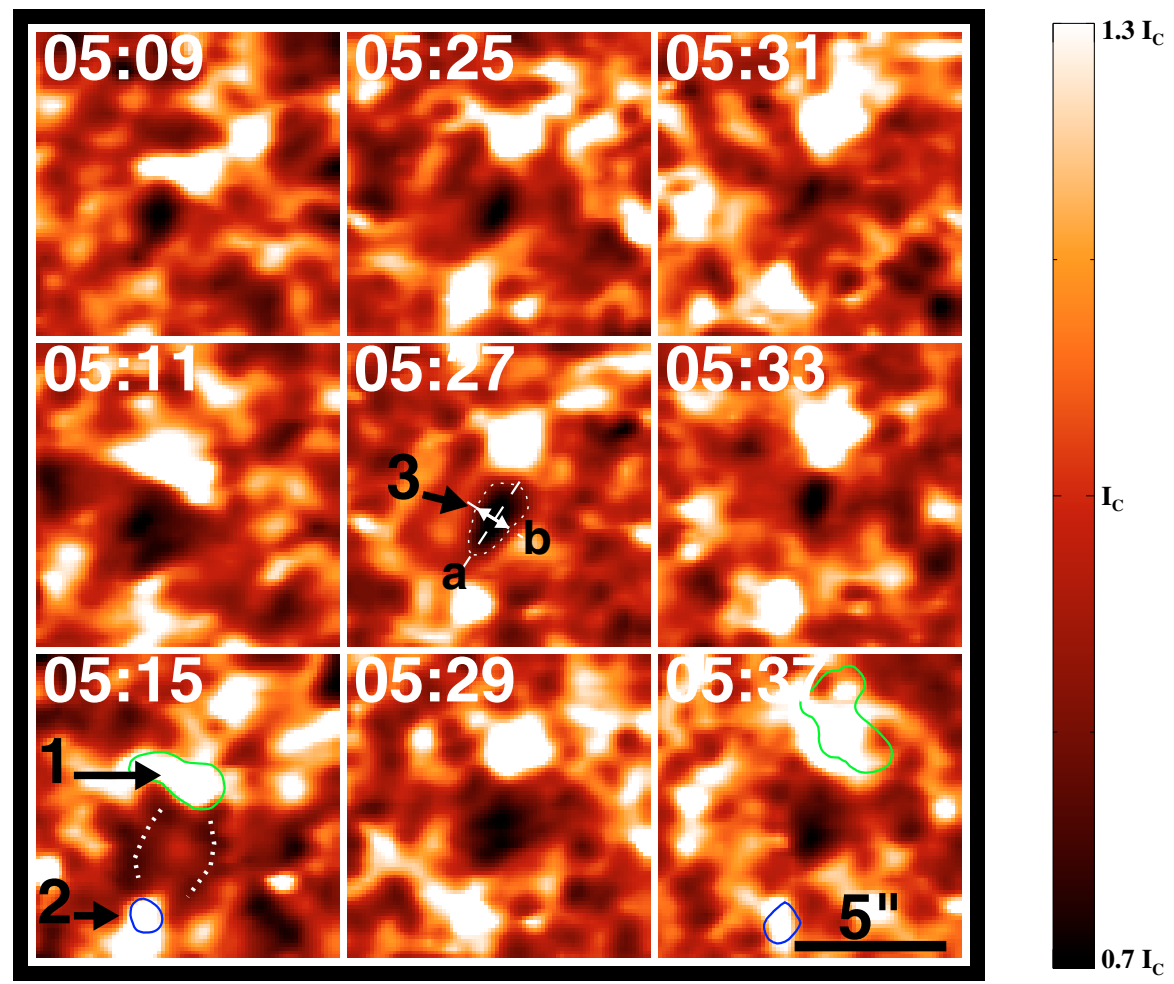

Fig. 4. Sequence of $\mathrm{Ca}$ II $\mathrm{H}$ images showing three developing processes of three mini AFs led by the emergence of the new dipole (see Fig. 3). The dipolar elements (indicated by arrows " 1 " and " 2 ") at 05:15 UT and 05:49 UT have been contoured to the temporally nearest panels. The two thick dotted lines indicate two segments that divided from the initial one. Arrow " 3 " denotes the main body of the AF (described with the thin dotted line). Line "a" is drawn along a filament, while line "b" is perpendicular to line "a" and put at the midpoint of the intercept of "a" with the contour. Bi-head arrow expresses the width of the AF.

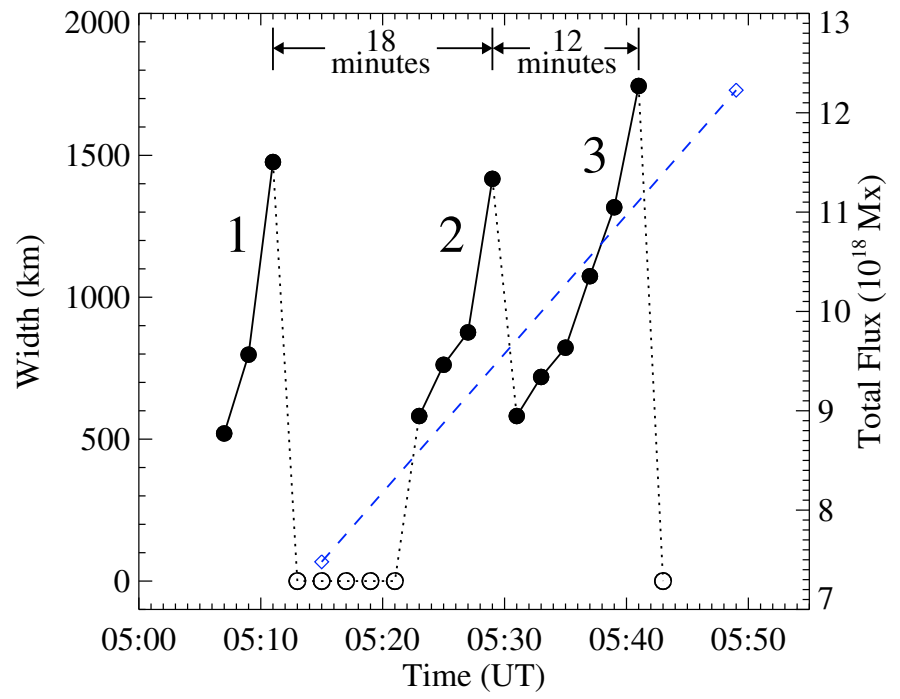

Fig. 5. Temporal variation of the width (circle symbols) of the AFs and the total unsigned magnetic flux (diamonds) of the dipole. The solid line "1" ("2"/“"3") connecting the filled circles represents one visible dividing (erupting) process, while dotted lines indicate the invisible stages. When the AF divided or no AF could be distinguished from the background, the width is set to zero (unfilled circles). The dashed line represents the change of the total unsigned magnetic flux.

brightness of the $\mathrm{Ca}$ II $\mathrm{H}$ line images and find it increased about 15\% at 03:21 UT compared to that at 01:45 UT, exhibiting an increasing trend.

\subsection{Magnetic flux cancellation at the $\mathrm{CH}$ boundary}

Magnetic flux cancellation is another primary form of magnetic field evolution. In region "III" (see Fig. 1) located at the boundary of the $\mathrm{CH}$, we observe the flux cancellations that occurred between one group of negative NT elements and two groups of positive ones in turn, as shown in Fig. 7. The left column shows a time sequence of magnetic field evolution. Dashed lines represent the $\mathrm{CH}$ boundary. The negative elements (outlined with octagons), initially located in the $\mathrm{CH}$, moved toward the boundary and partially cancelled with the positive elements (outlined with ellipses of thick lines) outside the $\mathrm{CH}$. At 05:02 UT, the negative elements had become much smaller. They encountered another positive cluster (outlined with ellipses of thin lines) and interacted with it. The middle column images show the corresponding response of the $\mathrm{Ca}$ II $\mathrm{H}$ line, and the right column the $171 \AA$ line. We can see that the bright points of $\mathrm{Ca}$ II $\mathrm{H}$ coincide well with the magnetic NT elements in location, while the brighter regions seen in the $171 \AA$ images only appear to coincide with the underlying magnetic elements when they are interacting.

As indicated by arrows " 1 " and " 2 " at 03:21 UT and 05:36 UT, much stronger cancellations took place with more obvious brightening of the upper atmosphere. The $171 \AA$ brightness in the cancelling areas kept increasing during the two cancelling phases. At 03:21 UT and 05:36 UT, it increased respectively about $10 \%$ and $8 \%$ over that of the pre-cancellation phase.

\subsection{Magnetic flux distribution in the $\mathrm{CH}$}

Besides the above case studies of the atmospheric response to the magnetic field evolution, we have investigated the relations between the distribution of magnetic flux and brightness at different atmospheric layers in the $\mathrm{CH}$ (the common part of the $\mathrm{SP}$ and BFI FOVs). We measure the magnetic flux of each NT and IN element in the LOS magnetogram obtained from 04:51 UT to 05:23 UT and the brightness of the corresponding images of several spectral lines. In order to compare the results of both negative and positive elements, two kinds are measured separately. For the NT fields, 66 negative and 14 positive elements were identified and measured, and the total magnetic flux is $-1.85 \times 10^{20} \mathrm{Mx}$ and $0.21 \times 10^{20} \mathrm{Mx}$, respectively. We also 


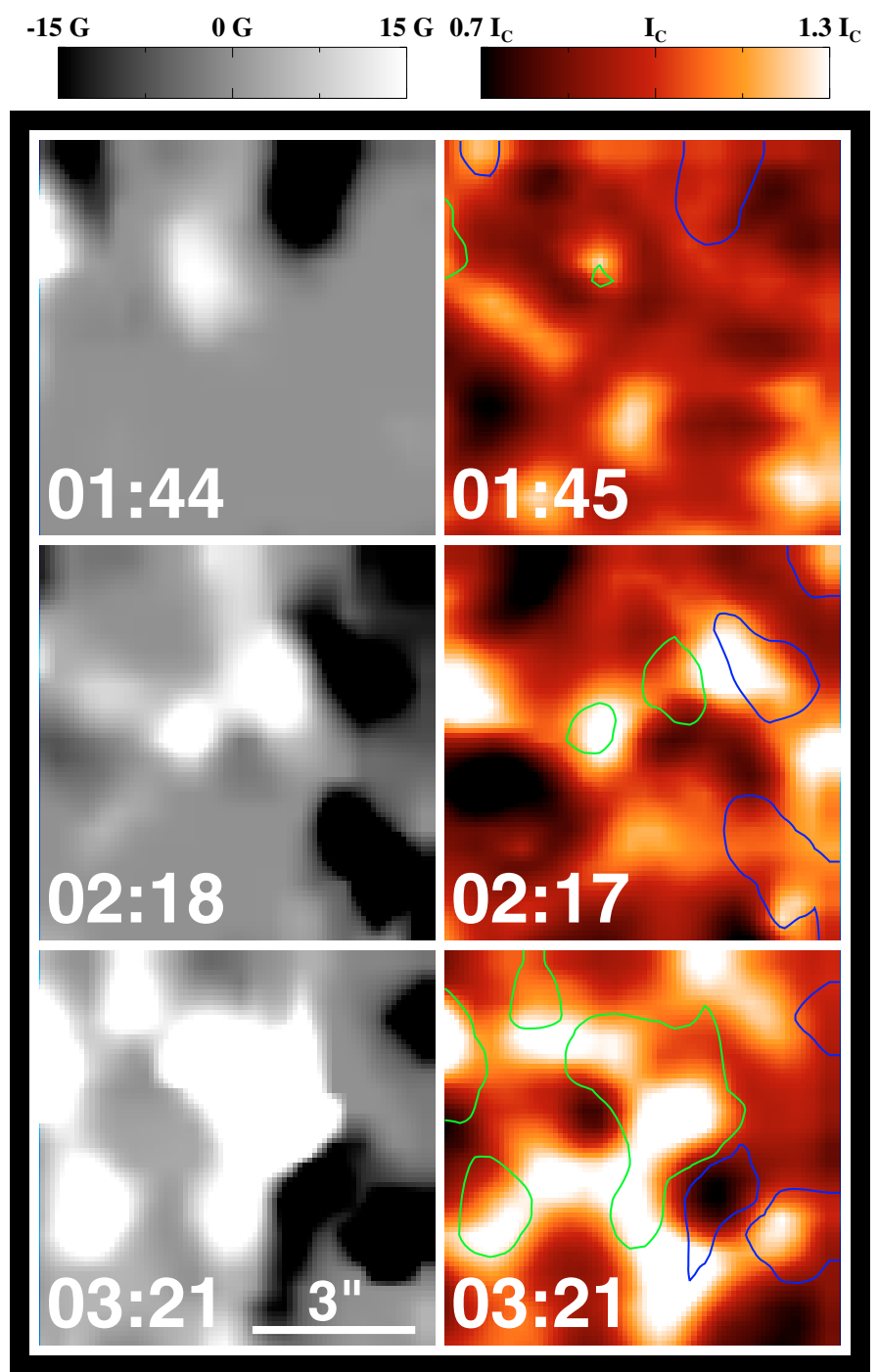

Fig. 6. Example of IN flux emergence of mixed-polarity clusters (region "II" in Fig. 1). The left column shows LOS magnetograms, while the right column consists of $\mathrm{Ca}$ II $\mathrm{H}$ images. Green and blue contours represent the positive and negative elements at $+15 \mathrm{G}$ and $-15 \mathrm{G}$ levels, respectively.

measured 593 negative and 571 positive IN elements, and the total flux is $-0.97 \times 10^{20} \mathrm{Mx}$ and $0.78 \times 10^{20} \mathrm{Mx}$, respectively. Then we deduce the average magnetic flux density and brightness of each element.

Figure 8 shows the relationship between the normalized brightness and the magnetic flux densities. The large (small) red and black dots represent the positive and negative NT (IN) elements, respectively. Figure 8 a demonstrates that there exists a high positive correlation between the $\mathrm{G}$ band brightness and the magnetic flux density for the NT elements. If two polarities are considered together, the linear correlation for the NT will be as high as 0.85 . We also calculate the linear correlation coefficient for the IN and find it is only 0.27 . Figure $8 \mathrm{~b}$ exhibits a linear correlation for the NT of the $\mathrm{Ca}$ II $\mathrm{H}$ line, only distinguished from that of the $\mathrm{G}$ band by the lower correlation coefficient (0.58). But neither the positive nor the negative IN has this type of trend. Figures 8c,d display no correlation between the $304 \AA$ ( $171 \AA$ ) brightness and the magnetic flux densities for both the NT and the IN. The results of $195 \AA$ and $284 \AA$ are similar to Figs. $8 \mathrm{c}-\mathrm{d}$.

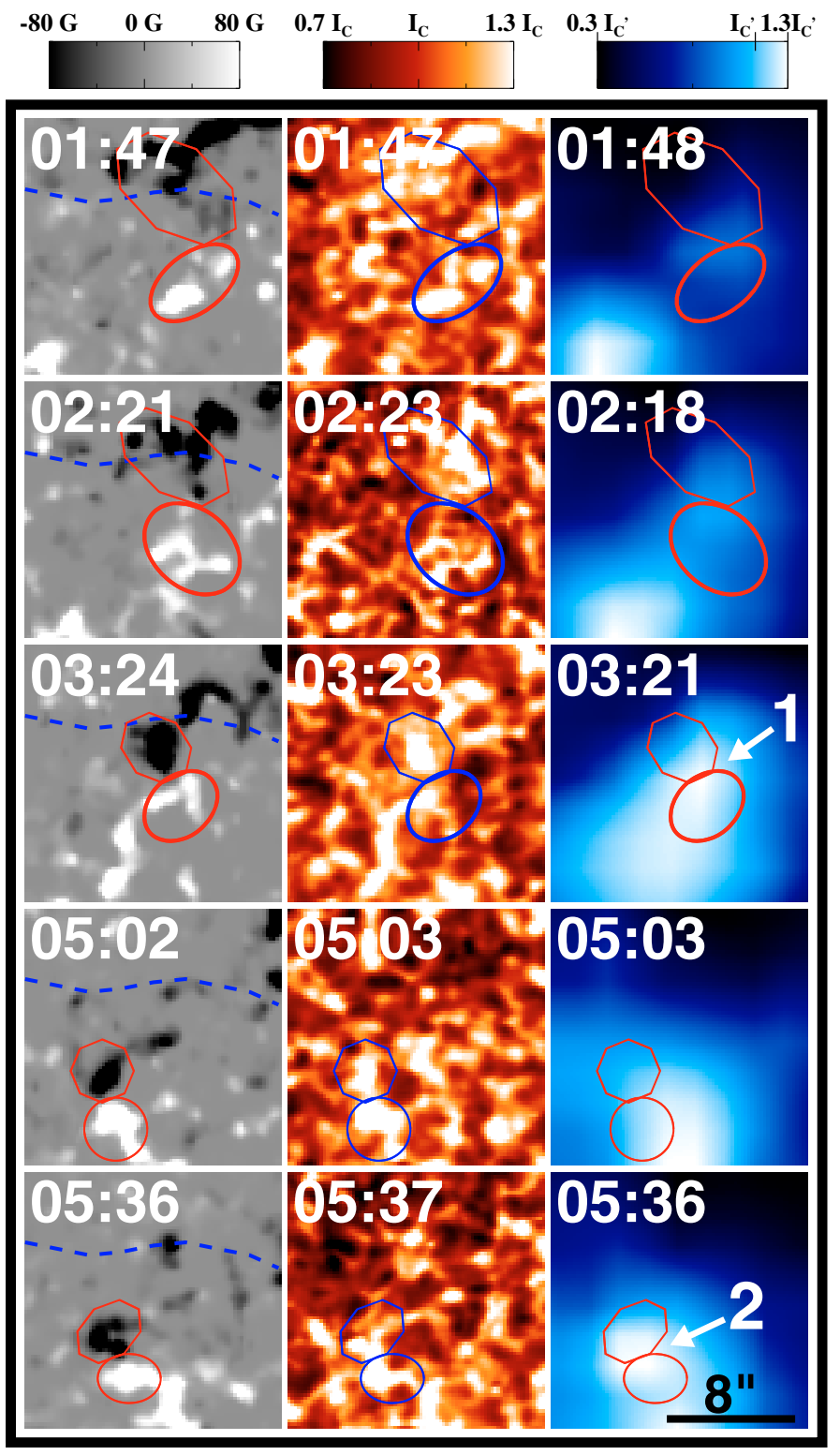

Fig. 7. Example of NT flux cancellation located at the $\mathrm{CH}$ boundary (region "III" in Fig. 1). The time sequence of LOS magnetograms (left column) shows the cancellations between one group of negative elements (outlined with octagons) and two groups of positive elements (outlined with ellipses of thick and thin line) during their evolution. Dashed lines represent the boundary of the $\mathrm{CH}$. The middle column images are the corresponding response of the Ca II $\mathrm{H}$ line, right column that of $171 \AA$ line. Note that the adjacent areas of the octagons and the ellipses are the places where the cancellations occur. Arrows " 1 " and " 2 " indicate the areas where much stronger cancellations take place. The $\mathrm{I}_{\mathrm{c}^{\prime}}$ represents the average value of $171 \AA$ intensities in the full-disk of $171 \AA$ images.

\section{Conclusions and discussion}

We have investigated an equatorial $\mathrm{CH}$ and its boundary region observed simultaneously by HINODE and STEREO on July 27, 2007. With the help of the vector magnetic fields and the Dopplergrams, we studied an emerging dipole located at the $\mathrm{CH}$ boundary. Three AFs accompanying the dipole appeared and expanded in turn, observed with the $\mathrm{Ca}$ II $\mathrm{H}$ line. The first $\mathrm{AF}$ divided into two segments in its late stage. The second and third AFs erupted and diffused with extremely rapidly in their late stages. The lifetimes of these three AFs are 4, 6, $10 \mathrm{~min}$, and 

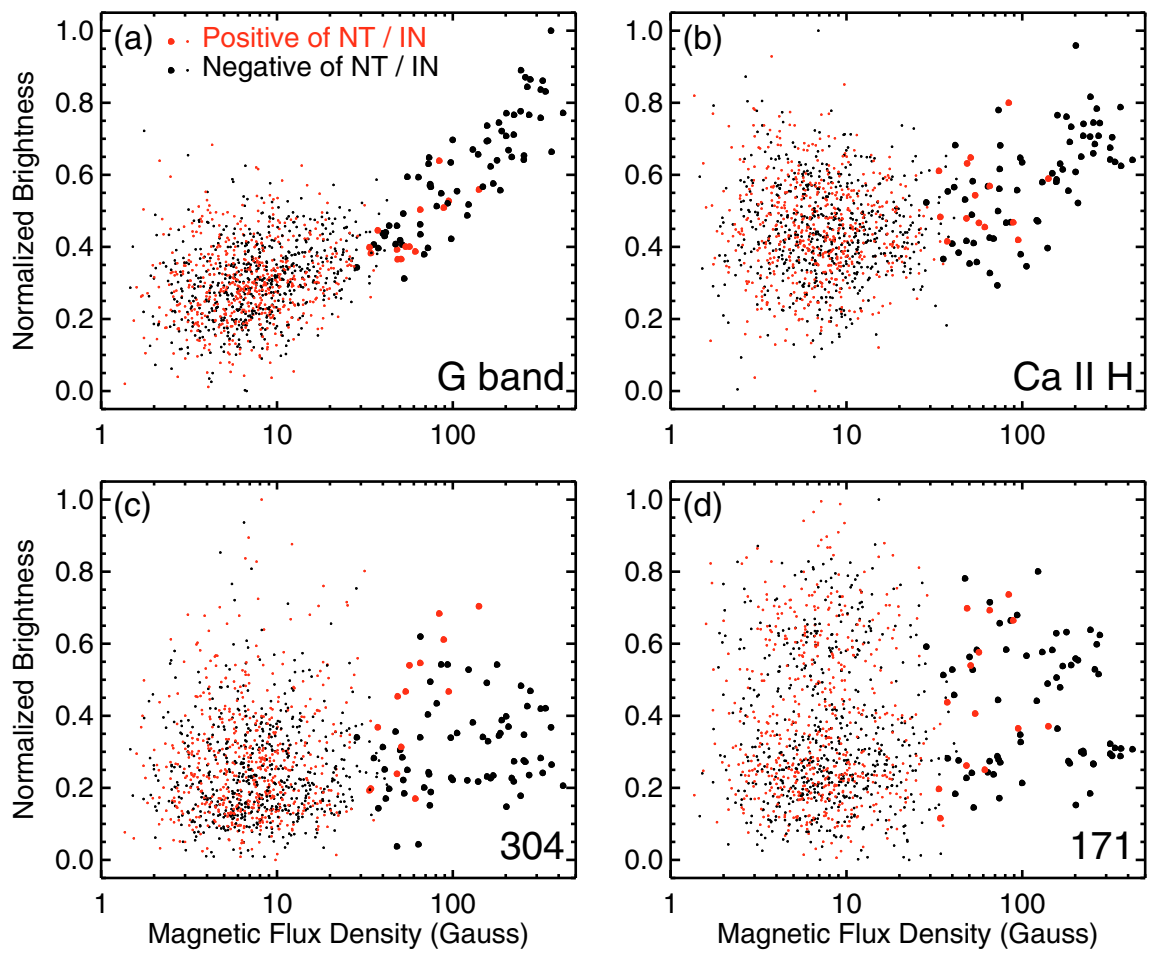

Fig. 8. Relationship between the normalized brightness of the images of different wavelengths and the absolute values of the magnetic flux densities. The large (small) red and black dots represent the positive and negative data of the NT (IN) elements, respectively.

the two intervals between the three divisions or eruptions are 18 and $12 \mathrm{~min}$. We display an example of mixed-polarity flux emergence of IN fields within the $\mathrm{CH}$ and present the corresponding chromospheric response. With the increase of the integrated magnetic flux, the brightness of the $\mathrm{Ca}$ II $\mathrm{H}$ images shows an increasing trend. In addition, we also study magnetic flux cancellations of NT fields located at the $\mathrm{CH}$ boundary and present the chromospheric and coronal response. By examining the magnetic NT and IN elements and the response of different atmospheric layers, we find there exists a close positive linear correlation (correlation coefficient 0.8 ) between the $\mathrm{G}$ band brightness and the magnetic flux density for the NT. The correlation coefficient for the IN of the $\mathrm{G}$ band is much lower, only 0.27 . We also obtain a close linear correlation ( correlation coefficient 0.58 ) for the NT of the Ca II H line.

In order to illustrate the intermittent appearance and eruptions of the AFs, a series of diagrams is provided (see Fig. 9), based on the previous model provided by Frazier (1972). The process of multi-eruptions can be decomposed into four phases (Figs. 9a-d). Dashed lines indicate the lower limit where the Ca II $\mathrm{H}$ line is formed. In Fig. 9a, there is no visible AF because all the magnetic flux tubes are too low to be observed with the Ca II $\mathrm{H}$ line. Then the tubes rise and part of flux tube " 1 " begins to be observed as an AF (darker part) (Fig. 9b). Tube "1" continues to rise and expand, so the AF apparently becomes bigger (Fig. 9c). In the late stage (Fig. 9d), the AF erupts suddenly and becomes optically thin and invisible instantly, within the visible region of the $\mathrm{Ca}$ II $\mathrm{H}$ line, following which another $\mathrm{AF}$ develops. In each phase, the dipolar footpoints persistently remain separate, accompanied by new flux emerging.

Yamauchi et al. (2005) have studied the eruptions of minifilaments, running nearly parallel to the neutral lines and crossing them with small angles, in a $\mathrm{CH}$. A filament erupted and rose to a high level where its top was invisible. Then a new visible filament formed along the initial path of the erupted one. Similarly, rising rapidly to a height far above the layer where the
Ca II $\mathrm{H}$ line was formed may be the cause of the invisibility of AFs in their late stages reported in this paper, whether they are optically thin or not.

$\mathrm{CH}$ boundaries separate the $\mathrm{CH}$ s from the surrounding quiet regions and play crucial roles in the evolution of the $\mathrm{CHs}$. Kahler \& Hudson (2002; see also Dahlburg \& Einaudi 2003) have researched the magnetic morphology of $\mathrm{CH}$ boundaries. These studies are helpful to understand the rigid rotation which is most likely correlated with the processes occurring at the boundaries (Nash et al. 1988; Wang \& Sheeley 1990, 2004; Fisk et al. 1999; Fisk \& Schwadron 2001). In order to maintain the integrity of the $\mathrm{CHs}$, magnetic reconnection must occur continuously at the boundaries (Wang et al. 1996; Kahler \& Hudson 2002). Wang et al. (1996) and Schwadron et al. (1999) suggested that there exists magnetic reconnection between the open field lines and the adjacent closed ones along the boundaries. Evidence for such reconnection was obtained with the demonstration of bidirectional jets by Madjarska et al. (2004) for the first time. Then more direct evidence was given by Raju et al. (2005) and Baker et al. (2007). When magnetic reconnection occurs, the magnetic field is restructured, accompanied by energy release (e.g. bright point appears in the EUV image); meanwhile, a small loop forms and becomes submerged leading to an observational phenomenon magnetic flux cancellation, as shown in Fig. 7 (see also Wang \& Shi 1993; Zhang et al. 2001, 2007b).

Our result that there are close positive linear correlations between the NT magnetic flux densities and the brightness of both $\mathrm{G}$ band and $\mathrm{Ca}$ II $\mathrm{H}$ in the $\mathrm{CH}$ is consistent with the research of Zirin (1988) and Nindos \& Zirin (1998) in the QS. The lower correlation for the NT of the Ca II H than of the G band can be considered to be caused by the fact that, when the flux tubes extend to the chromosphere, they expand, becoming larger than in the photosphere. With increased height, the flux tubes enlarge and most of them change direction. Especially in the corona, the average expansion factor (ratio of the maximum to the minimum of the flux tube area) is $28 \pm 11$ (Wiegelmann et al. 2005). The 
(a) lines are mainly due to the lower tempo-spatial resolution of the images and the incapacity of the IN flux tubes to extend into the transition region and the corona (Wiegelmann \& Solanki 2004; Ca II
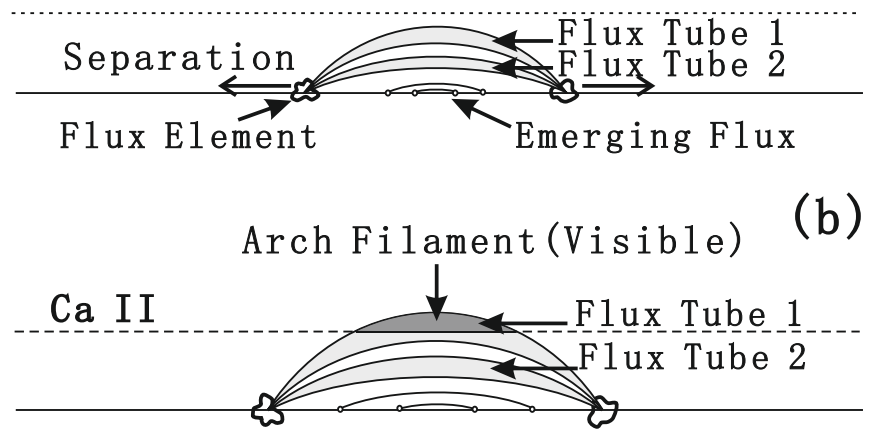

(c)
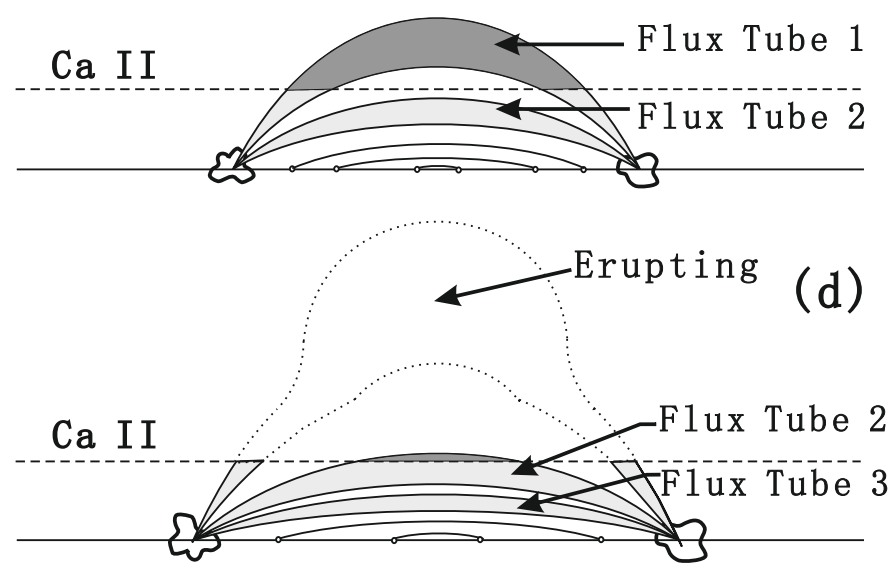

Fig. 9. Multi-eruption process of the small-scale AFS. Dashed lines indicate the lower limit where the Ca II $\mathrm{H}$ line is formed. In phase "a", there is no visible AF because all the magnetic flux tubes are too low to be observed with the $\mathrm{Ca}$ II $\mathrm{H}$ line. Then the tubes rise and part of flux tube 1 begins to be observed as an AF (darker part), as shown in phase "b". From phase "b" to phase "c", tube 1 continues to rise and expand, so the AF apparently becomes larger. In the late stage (phase "d"), the AF erupts suddenly and becomes optically thin and invisible within the visible region of the $\mathrm{Ca}$ II $\mathrm{H}$ line, following which another developing and eruption process for flux tube 2 begins.

decline and expansion of the flux tubes can explain why there is no correlation between the transition region and the corona (exhibited by Figs. $8 \mathrm{c}$ and 8d), although the relatively lower tempospatial resolution of the images from STEREO may be one cause.

A large part of scatter of the data points for the IN of the $\mathrm{Ca}$ II $\mathrm{H}$ in Fig. $8 \mathrm{~b}$ is mainly due to the 3 -min oscillations, which does not allow us to obtain a correlation between the brightness and magnetic fields even if such a correlation exists on the Sun (Sivaraman et al. 2000). The low correlation coefficient (0.27) for the IN of the G band in Fig. 8a also may be affected by the 3 -min oscillations. Another reason for the lack of an obvious linear correlation for both the G band and Ca II H IN may be that the cadence of $\mathrm{G}$ band and $\mathrm{Ca}$ images is not high enough. The average lifetime of the IN elements is only about $4.84 \mathrm{~min}$ (Zhou, G. private communication), so many of the magnetic IN elements have evolved noticeably within one minute. This can also explain why there is only partial coincidence between the magnetic elements and the bright features shown in Fig. 6. The poor correlations for the IN of $304 \AA, 171 \AA, 195 \AA$ and $284 \AA$
Wiegelmann et al. 2005).

Acknowledgements. We are grateful to the HINODE, STEREO and SOHO teams for providing the data. HINODE is a Japanese mission developed and launched by ISAS/JAXA, with NAOJ as the domestic partner and NASA and STFC (UK) as international partners. It is operated by these agencies in co-operation with ESA and NSC (Norway). This work is supported by the National Natural Science Foundations of China (G40674081, 40890161, 10573025, 10703007, and 10733020), the CAS Project KJCX2-YW-T04, and the National Basic Research Program of China under grant G2006CB806303.

\section{References}

Akinari, N. 2007, ApJ, 660, 1660

Alissandrakis, C. E., Tsiropoula, G., \& Mein, P. 1990, A\&A, 230, 200

Baker, D., van Driel-Gesztelyi, L., \& Attrill, G. D. R. 2007, Astron. Nachr., 328, 773

Beck, C., Schmidt, W., Rezaei, R., \& Rammacher, W. 2008, A\&A, 479, 213

Belenko, I. A. 2001, Sol. Phys., 199, 23

Bilenko, I. A. 2002, A\&A, 396, 657

Bilenko, I. A. 2004, Sol. Phys., 221, 261

Bohlin, J. D. 1977, Sol. Phys., 51, 377

Bruzek, A. 1967, Sol. Phys., 2, 451

Bruzek, A. 1969, Sol. Phys., 8, 29

Carlsson, M., Stein, R. F., Nordlund, Å., \& Scharmer, G. B. 2004, ApJ, 610, L137

Chou, D. Y. 1993, ed. Zirin, H., Ai, P., \& Wang, H., ASP Conf. Ser. 46, 471

Chou, D. Y., \& Zirin, H. 1988, ApJ, 333, 420

Cranmer, S. R. 2002, Space Sci. Rev., 101, 229

Crooker, N. U., \& Cliver, E. W. 1994, J. Geophys. Res., 99, 23383

Dahlburg, R. B., \& Einaudi, G. 2003, Adv. Space Res., 32, 1125

Delaboudinière, J. P., Artzner, G. E., Brunaud, J., et al. 1995, Sol. Phys., 162, 291

Domingo, V., Fleck, B., \& Poland, A. I. 1995, Sol. Phys., 162, 1

Dowdy, J. F. 1993, ApJ, 411, 406

Dowdy, J. F., Rabin, D., \& Moore, R. L. 1986, Sol. Phys., 105, 35

Dwivedi, B. N., \& Srivastava, A. K. 2006, Sol. Phys., 237, 143

Feldman, U., \& Laming, J. M. 2000, Phys. Scr, 61, 222

Feldman, U., Dammasch, I. E., \& Wilhelm, K. 2001, ApJ, 558, 423

Fisk, L. A., \& Schwadron, N. A. 2001, ApJ, 560, 425

Fisk, L. A., Zurbuchen, T. H., \& Schwadron, N. A. 1999, ApJ, 521, 868

Frazier, E. N. 1970, Sol. Phys., 14, 89

Frazier, E. N. 1972, Sol. Phys., 26, 130

Georgaklilas, A. A., Alissandrakis, C. E., \& Zachariadis, T. G. 1990, Sol. Phys., 129,277

Giordano, S., Antonucci, E., Noci, G., et al. 2000, ApJ, 531, L79

Hackenberg, P., Marsch, E., \& Mann, G. 2000, A\&A, 360, 1139

Harvey, J. W., \& Sheeley, N. R. 1979, Space Sci. Rev., 23, 139

Harvey, K. L. 1996, in Gamma-ray bursts: 3rd Huntsville Symposium, AIP Conf. Proc., 382, 9

Harvey, K. L., \& Recely, F. 2002, Sol. Phys., 211, 31

Hofer, M. Y., \& Storini, M. 2002, Sol. Phys., 207, 1

Howard, R. A., Moses, J. D., Vourlidas, A., et al. 2008, Space Sci. Rev., 136, 67

Ichimoto, K., Lites, B., Elmore, D., et al. 2008, Sol. Phys., 249, 233

Janse, Å. M., Lie-Svendsen, Ø., \& Leer, E. 2007, A\&A, 474, 997

Jordan, C., Macpherson, K. P., \& Smith, G. R. 2001, MNRAS, 328, 1098

Kahler, S. W., \& Hudson, H. S. 2002, ApJ, 574, 467

Kaiser, M. L., Kucera, T. A., Davila, J. M., et al. 2008, Space Sci. Rev., 136, 5

Kobanov, N. I., \& Sklyar, A. A. 2007, Astron. Rep., 51, 773

Kobanov, N. I., Makarchik, D. V., \& Sklyar, A. A. 2003, Sol. Phys., 217, 53

Kosugi, T., Matsuzaki, K., Sakao, T., et al. 2007, Sol. Phys., 243, 3

Krieger, A. S., Timothy, A. F., \& Roelof, E. C. 1973, Sol. Phys., 29, 505

Laming, J. M., \& Feldman, U. 2003, ApJ, 591, 1257

Leighton, R. B. 1959, ApJ, 130, 366

Leighton, R. B., Noyes, R. W., \& Simon, G. W. 1962, ApJ, 135, 474

Levine, R. H. 1977, ApJ, 218, 291

Lites, B. W., Elmore, D. F., \& Streander, K. V. 2001, in Advanced Solar Polarimetry-Theory, Observation, and Instrumentation, ed. M. Sigwarth (San Francisco: ASP), ASP Conf. Ser. 236, 33

Lites, B. W., Rutten, R. J., \& Berger, T. E. 1999, ApJ, 517, 1013

Livingston, W. C., \& Harvey, J. 1975, BAAS, 7, 346

Madjarska, M. S., Doyle, J. G., \& van Driel-Gesztelyi, L. 2004, ApJ, 603, L57

Mahajan, S. M., Miklaszewski, R., Nikol'Skaya, K. I., \& Shatashvili, N. L. 2002, Adv. Space. Res., 30, 545 
Maravilla, D., Lara, A., Valdés Galicia, J. F., \& Mendoza, B. 2001, Sol. Phys., 203, 27

Markovskii, S. A., \& Hollweg, J. V. 2004, ApJ, 609, 1112

Meunier, N. 2005, A\&A, 443, 309

Moran, T. G. 2003, ApJ, 598, 657

Munro, R. H., \& Withbroe, G. L. 1972, ApJ, 176, 511

Nash, A. G., Sheeley, N. R., \& Wang, Y. M. 1988, Sol. Phys., 117, 359

Nindos, A., \& Zirin, H. 1998, Sol. Phys., 179, 253

O'Shea, E., Banerjee, D., \& Doyle, J. G. 2005, A\&A, 436, L35

O'Shea, E., Banerjee, D., \& Doyle, J. G. 2006, A\&A, 452, 1059

O'Shea, E., Banerjee, D., \& Doyle, J. G. 2007, A\&A, 463, 713

Patsourakos, S., \& Vial, J. C. 2000, A\&A, 359, L1

Patsourakos, S., Habbal, S. R., \& Hu, Y. Q. 2002, ApJ, 581, L125

Raju, K. P., Bromage, B. J. I., Chapman, S. A., \& Del Zanna, G. 2005, A\&A, 432, 341

Raju, K. P., Sakurai, T., Ichimoto, K., \& Singh, J. 2000, ApJ, 543, 1044

Reeves, E. M., \& Parkinson, W. H. 1970, ApJS, 21, 1

Reeves, E. M., Timothy, J. G., \& Huber, M. C. E. 1977, Appl. Opt., 16, 837

Remling, B., Deubner, F. L., \& Steffens, S. 1996, A\&A, 316, 196

Rezaei, R., Schlichenmaier, R., Beck, C. A. R., et al. 2007, A\&A, 466, 1131

Scherrer, P. H., Bogart, R. S., Bush, R. I., et al. 1995, Sol. Phys., 162, 129

Schwadron, N. A., Fisk, L. A., \& Zurbuchen, T. H. 1999, ApJ, 521, 859

Shen, C. L., Wang, Y. M., Ye, P. Z., \& Wang, S. 2006, ApJ, 639, 510

Shimizu, T., Nagata, S., Tsuneta, S., et al. 2008, Sol. Phys., 249, 221

Sivaraman, K. R., \& Livingston, W. C. 1982, Sol. Phys., 80, 227

Sivaraman, K. R., Gupta, S. S., Livingston, W. C., et al. 2000, A\&A, 363, 279

Skumanich, A., Smythe, C., \& Frazier, E. N. 1975, ApJ, 200, 747

Srivastava, A. K., \& Dwivedi, B. N. 2007, JA\&A, 28, 1

Steffens, S., Hofmann, J., \& Deubner, F. L. 1996, A\&A, 307, 288

Stenflo, J. O., \& Harvey, J. W. 1985, Sol. Phys., 95, 99

Stepanian, N. N. 1995, Izv. Ross. Akad. Nauk, Ser. Fiz., 59(7), 63

Stucki, K., Solanki, S. K., Schühle, U., et al. 2000, A\&A, 363, 1145

Stucki, K., Solanki, S. K., Pike, C. D., et al. 2002, A\&A, 381, 653

Suematsu, Y., Tsuneta, S., Ichimoto, K., et al. 2008, Sol. Phys., 249, 197

Temmer, M., Vršnak, B., \& Veronig, A. M. 2007, Sol. Phys., 241, 371

Teplitskaya, R. B., Turova, I. P., \& Ozhogina, O. A. 2007, Sol. Phys., 243, 143

Teriaca, L., Poletto, G., Romoli, M., \& Biesecker, D. A. 2003, ApJ, 588, 566

Tian, H., Marsch, E., Tu, C. Y., et al. 2008, A\&A, 482, 267

Tsiropoula, G., Georgakilas, A. A., Alissandrakis, C. E., \& Mein, P. 1992, A\&A, 262,587

Tsuneta, S., Ichimoto, K., Katsukawa, Y., et al. 2008, Sol. Phys., 249, 167

Tsurutani, B. T., \& Gonzalez, W. D. 1987, Planet. Space Sci., 35, 405
Tu, C. Y., Zhou, C., Marsch, E., et al. 2005, Sci, 308, 519

Underwood, J. H., \& Muney, W. S. 1967, Sol. Phys., 1, 129

Vernazza, J. E., Avrett, E. H., \& Loser, R. 1981, ApJS, 45, 635

Veronig, A. M., Temmer, M., Vršnak, B., \& Thalmann, J. K. 2006, ApJ, 647, 1466

Vršak, B., Temmer, M., \& Veronig, A. M. 2007, Sol. Phys., 240, 315

Waldmeier, M. 1951, ZAp, 30, 1

Wang, J. X., \& Shi, Z. X. 1993, Sol. Phys., 143, 119

Wang, Y. M., \& Sheeley, N. R. 1990, ApJ, 365, 372

Wang, Y. M., \& Sheeley, N. R. 2004, ApJ, 612, 1196

Wang, Y. M., Hawley, S. H., \& Sheeley, N. R. 1996, Sci, 271, 464

Warren, H. P., \& Winebarger, A. R. 2000, ApJ, 535, L63

Weart, S. R. 1970, ApJ, 162, 987

Wiegelmann, T., \& Solanki, S. K. 2004, Sol. Phys., 225, 227

Wiegelmann, T., Xia, L. D., \& Marsch, E. 2005, A\&A, 432, L1

Wilhelm, K. 2006, A\&A, 455, 697

Wilhelm, K., Dammasch, I. E., Marsch, E., \& Hassler, D. M. 2000, A\&A, 353, 749

Wilhelm, K., Dammasch, I. E., \& Xia, L. D. 2002, Adv. Space Res., 30, 517

Worden, J., Harvey, J., \& Shine, R. A. 1999, ApJ, 523, 450

Wu, D. J., \& Yang, L. 2007, ApJ, 659, 1693

Xia, L. D., Marsch, E., \& Curdt, W. 2003, A\&A, 399, L5

Xia, L. D., Marsch, E., \& Wilhelm, K. 2004, A\&A, 424, 1025

Yamauchi, Y., Moore, R. L., Suess, S. T., et al. 2004, ApJ, 605, 511

Yamauchi, Y., Wang, H., Jiang, Y., et al. 2005, ApJ, 629, 572

Zhang, J., Lin, G. H., Wang, J. X., et al. 1998a, A\&A, 338, 322

Zhang, J., Lin, G. H., Wang, J. X., et al. 1998b, Sol. Phys., 178, 245

Zhang, J., Wang, J. X., Wang, H. M., \& Zirin, H. 1998c, A\&A, 335, 341

Zhang, J., Wang, J. X., Deng, Y. Y., \& Wu, D. J. 2001, ApJ, 548, L99

Zhang, J., Woch, J., Solanki, S. K., \& von Steiger, R. 2002, Geophys. Res. Lett., 29, 1236

Zhang, J., Woch, J., Solanki, S. K., et al. 2003, J. Geophys. Res., 108, 1144

Zhang, J., Woch, J., \& Solanki, S. 2005a, ChJAA, 5, 531

Zhang, J., Ma, J., \& Wang, H. M. 2006, ApJ, 649, 464

Zhang, J., Zhou, G. P., Wang, J. X., \& Wang, H. M. 2007a, ApJ, 655, L113

Zhang, J., Li, L. P., \& Song, Q. 2007b, ApJ, 662, L35

Zhang, T. X. 2003, ApJ, 597, L69

Zhang, T. X., Wang, J. X., \& Xiao, C. J. 2005b, ChJAA, 5, 285

Zirin, H. 1988, Astrophysics of the Sun (Cambridge: Cambridge University Press)

Zirker, J. B., ed. 1977, Coronal Holes and High-Speed Wind Streams (Boulder: Colorado Associated Univ. Press) 